\title{
CD276 suppresses CAR-T cell function by promoting tumor cell glycolysis in esophageal squamous cell carcinoma
}

\author{
Guangxing Yue ${ }^{1}$, Jingwen Tang ${ }^{1}$, Lihan Zhang ${ }^{1}$, Hong Niu ${ }^{1}$, Huahua Li $^{1}$, Suxia Luo ${ }^{2}$ \\ ${ }^{1}$ Department of Integrated Chinese and Western Medicine, Affiliated Cancer Hospital of Zhengzhou University \& Henan Cancer Hospital, \\ Zhengzhou, China; ${ }^{2}$ Department of Medicine Oncology, Affiliated Cancer Hospital of Zhengzhou University, Zhengzhou, China \\ Contributions: (I) Conception and design: G Yue, S Luo; (II) Administrative support: J Tang, G Yue; (III) Provision of study materials or patients: L \\ Zhang; (IV) Collection and assembly of data: H Niu; (V) Data analysis and interpretation: H Li; (VI) Manuscript writing: All authors; (VII) Final \\ approval of manuscript: All authors. \\ Correspondence to: Suxia Luo. Department of Medicine Oncology, Affiliated Cancer Hospital of Zhengzhou University, Zhengzhou 450052, China. \\ Email: zlyyluosuxia0361@zzu.edu.cn.
}

Background: As an immune checkpoint that suppresses antitumor immunity, CD276 is a potential therapeutic target for cancer immunotherapy. However, the role of CD276 in esophageal squamous cell carcinoma (ESCC) has not been thoroughly examined. A greater understanding of the regulatory mechanism of CD276 may improve the clinical response and efficacy of cancer immunotherapy.

Methods: The expression of CD276 was measured by qRT-PCR, IHC and flow cytometry analysis. T cell infiltration in ESCC was measured by qRT-PCR and immunofluorescence analysis. The regulation function of CD276 in glucose metabolism was examined by metabolism assays, western blotting and small molecule inhibitors. Transfection was used for gene editing. The oncogenic function of CD276 was examined in vivo by CAR-T cell therapy model.

Results: Based on our findings, CD276 regulated the expression of the PKM2 gene in ESCC. Overexpression of CD276 induced the phosphorylation of PKM2 by the STAT3 signalling pathway to promote glucose metabolism in tumors. The accumulation of lactic acid in the tumor microenvironment has been reported to regulate the immune cells, particularly CD8+ T cells. We further analyzed the effect of CD276 on the function of T cells. Chimeric antigen receptor T cells (CAR-T) targeting human epidermal growth factor receptor 2 (HER2) were used as effector cells to detect the effect of CD276 on immunotherapy. The therapeutic effects of CAR-T cells were markedly limited by CD276 overexpression.

Conclusions: Our results are the first to show that tumor-derived CD276 supports disease progression. Overexpression of CD276 promoted glucose metabolism in tumor and inhibited the function of CD8+ T cells. Therefore, strategies targeting CD276 might improve the response to cancer immunotherapy of ESCC patients.

Keywords: CD276; esophageal squamous cell carcinoma (ESCC); PKM2; glucose; chimeric antigen receptor T cells (CAR-T)

Submitted Jan 11, 2021. Accepted for publication Feb 08, 2021.

doi: 10.21037/jgo-21-50

View this article at: http://dx.doi.org/10.21037/jgo-21-50

\section{Introduction}

Esophageal squamous cell carcinoma (ESCC), which accounts for over $90 \%$ of esophageal cancer cases worldwide, is a common type of malignant tumor (1).
Despite developments in radiotherapy and surgical therapy, the prognosis of patients with ESCC has not improved significantly, and the 5 -year survival rate is only $15-25 \%$ (2). Currently, immunotherapy, including immune checkpoint 
inhibitors (ICIs) and chimeric antigen receptor $\mathrm{T}$ cell (CAR-T) therapy, is a relatively new field in the treatment of ESCC and has achieved encouraging therapeutic effects. However, due to the side effects and limited effects of this treatment, there is an urgent need to understand these new mechanisms of resistance (3).

CD276 (also called B7H3), a member of the B7/CD28 superfamily, was found to be expressed at high levels in multiple tumor types (4). Various cell types, such as endothelial cells, immune cells and osteoblasts, have also been reported to exhibit positive expression of CD276 (5). An the coinhibitory function of CD276 in immune cells has also been observed (6). The expression of CD276 is correlated with worse disease outcomes, and as a signalling molecule, it may play key roles in cancer and immune regulation (7). According to existing reports, overexpression of CD276 is clearly associated with an advanced tumor stage and lymph node metastasis in patients with non-small cell lung cancer. This effect of CD276 depends on the increased secretion of interleukin-10, which is an important anti-inflammatory cytokine (8). The function of CD276 in promoting multiple myeloma progression mainly depend on the activation of Src/STAT3 signalling and the degradation of SOCS3 protein (9). It has also been used as a target of anticancer therapies in multiple tumor types; for example, studies by Theruvath and Du have confirmed the potential of CD276 as a target for CAR-T cell therapy $(10,11)$.

Despite the rapid progress in CD276 research, the regulatory mechanisms of CD276 in ESCC remain largely unknown. In our study, we explored the expression of CD276 in ESCC and correlation between CD276 and T cell infiltration. Our results demonstrated that CD276 was abnormally expressed in patients with ESCC and promoted disease progression. Furthermore, the role of CD276 depended on metabolic reprogramming. Overexpression of CD276 induced the phosphorylation of pyruvate kinase M2 (PKM2) by the STAT3 signalling pathway, to promote the accumulation of lactic acid in the tumor microenvironment. Moreover, a mouse model was used to confirm the inhibition of CD276 by CAR-T cell therapy. We present the following article in accordance with the MDAR reporting checklist (available at http://dx.doi.org/10.21037/ jgo-21-50).

\section{Methods}

\section{Bioinformatics data retrieval}

The Cancer Genome Atlas (TCGA) gene expression datasets of patients with ESCC were obtained (https:// tcgadata.nci.nih.gov/tcga/). Then, a differential gene expression analysis and immune-related gene analysis were executed based on these data. The Tumor Immune Estimation Resource 2.0 (TIMER2.0) (http://timer. cistrome.org/) was used to analyze the correlation of CD276 expression with the level of immune cell infiltration in tumor microenvironment (12).

\section{Clinical sample selection}

All fresh ESCC tissues were collected from patients during surgical excisions and divided into two separate tissue segments. One was used for further RNA extraction, and the other was embedded in paraffin. RNA was used for quantitative real-time polymerase chain reaction (qRT-PCR) and paraffin blocks were used for immunohistochemistry (IHC). The research protocol was reviewed and approved by the Ethics Committee of Zhengzhou University, and informed consent was obtained from all participants included in the study, in agreement with institutional guidelines (No. 2016-lw-107). This study conformed to the provisions of the Declaration of Helsinki (as revised in 2013).

\section{Cell culture and transfection}

Five ESCC cell lines (KYSE150, KYSE510, KYSE450, KYSE70, and EC109) were cultured in Dulbecco's Modified Eagle Medium (DMEM, Gibco) containing 5\% FBS (Gibco). T cells from human peripheral blood mononuclear cells (PBMCs) were cultured in RPMI-1640 (Gibco) supplemented with $10 \%$ FBS (Gibco). The lentivirus overexpression vector (pCDH-EF1a-CD276-GFP) or knockdown vector (pSI-H1-SHRNA-GFP) was used for tumor cell transfection. The lentivirus overexpression vector (pCDH-EF1a-CAR.Her2-GFP) was used for T cell transfection.

\section{Immunobistochemistry analysis}

Immunohistochemical staining was performed with Tissue Culture Staining Kit (Research And Diagnostic Systems, Inc.). Antibodies against human CD276, PKM2 and Ki67 were purchased from Cell Signaling Technology (CST). The staining intensity (scores: negative $=0$, weak $=1$, moderate $=2$, strong $=3$ ) and area extent (scores: $1-29 \%=1,30-69 \%=2,70-100 \%=3$ ) were scored using 
semiquantitative scoring criteria. Then, the two scores were multiplied to calculate the intensity of protein expression.

\section{Immunofluorescence analysis}

Antibodies against human CD276 and CD8 (CST) were used for immunofluorescence staining. Cells were blocked with $5 \%$ bovine serum albumin (BSA) and incubated with antibody for 2 hours at $4{ }^{\circ} \mathrm{C}$. For secondary antibody staining, cells were washed three times prior to a 60 minutes incubation with the secondary antibody at $4{ }^{\circ} \mathrm{C}$ in the dark. Microscopy images were captured using confocal microscope.

\section{$q R T-P C R$}

Total RNA extracted from ESCC tissues and ESCC cell lines was reverse transcribed into complement DNA (cDNA). The ViiA7 real-time PCR system (Thermo Fisher Scientific) was used to perform the qRT-PCR assay. The relative gene expression levels were normalized to GAPDH and calculated using the $2^{-\Delta \Delta C t}$ method (13). The primers used for amplification were synthesized by Shanghai Shengong Biotechnology Company and are listed in Table S1.

\section{Flow cytometry analysis}

Cell suspensions were harvested in cold phosphate-buffered saline (PBS) containing $0.5 \%$ vovine serum albumin (BSA), and then incubated with CD276-PE (BD Biosciences) for 30 minutes at $4{ }^{\circ} \mathrm{C}$. CD276 expression was analyzed using a FACSCanto Flow Cytometer (BD Biosciences). FlowJo-V10 software was used for further analyses.

\section{Western blotting}

An ultrasonic treatment was used for total protein extraction. The proteins were separated using SDS/ polyacrylamide gel electrophoresis and transferred to a nylon membrane. After blocking with Tris-buffered saline with Tween 20 (TBS-T) containing 5\% milk for 1 hour, the membranes were incubated with the primary antibodies (CST) overnight at $4{ }^{\circ} \mathrm{C}$. After washing with TBS-T, membranes were incubated with a secondary antibody (CST). Finally, antigen-antibody complexes were visualized with a chemiluminescence kit (Pierce).

\section{Metabolism assays}

Tumor cells were seeded in XFe 96-well microplates (6,000 cells/well) and incubated for 24 hours. Cells were washed and incubated in basal medium (Agilent Technologies) at $37^{\circ} \mathrm{C}$ for 1 hour. Real-time measurements of the mitochondrial extracellular acidification rate (ECAR) of tumor cells were performed using a SeahorseXFe96 analyzer (Seahorse Bioscience) according the manufacturer's instructions. Data were normalized to cell numbers that were measured using the YO-PRO®-1 Assay (Thermo Fisher Scientific).

\section{Lactate detection assay}

The culture supernatants of tumor cells exposed to different treatments were collected to detect lactate production, and a fresh culture medium was used as a control. The concentration of lactic acid secreted by tumor cells was measured using a lactic acid detection kit. The absorbance (OD $570 \mathrm{~nm}$ ) was measured with microplate reader. Lactate production was calculated from a standard curve.

\section{Animals and tumor cell subcutaneous implantation model}

All xenograft mouse model experiments were performed in the Henan Key Laboratory for Pharmacology of Liver Diseases, the animal experimentation certificate was approved by the ethics committee of Henan Key Laboratory for Pharmacology of Liver Diseases (approval number: 2019-41). All animal experiments were performed in accordance with the guidelines of ethics committee of Henan Key Laboratory for Pharmacology of Liver Diseases for animal use and care. Six-week-old female NOD/ SCID mice ordered from Vital River Laboratory Animal Technology Co. Ltd were housed in a specific pathogenfree environment. Tumor cells suspensions $\left(1 \times 10^{6}\right.$ cancer cells suspended in $100 \mu \mathrm{L}$ of PBS) were administered by subcutaneous injection, and T cell suspensions $\left(1 \times 10^{6}\right.$ cancer cells suspended in $100 \mu \mathrm{L}$ of PBS) were administered by a tail vein injection one week later. Changes in tumors were detected using an animal fluorescence imager. The tumor volume (volume $\mathrm{mm}^{3}=\mathrm{W}^{2} \times \mathrm{L} / 2$ ) and weight were recorded after the mice were sacrificed.

\section{Survival analysis}

Kaplan-Meier survival analysis was used to analyse the 
relationship between survival and CD276 expression in ESCC patients, and $\mathrm{P}<0.05$ was considered significant. The expression levels of the samples were grouped as high and low based on the cut-off point.

\section{Statistical analysis}

An unpaired Student's $t$-test (SPSS version 17.0, IBM Corp.) was conducted to determine the statistical significance of differences in the experimental data. A $\mathrm{P}$ value $<0.05$ was considered to indicate a significant difference between multiple groups. Except where otherwise indicated, experiments were repeated three times.

\section{Results}

\section{CD276 was upregulated in ESCC tissues and predicted poor prognosis}

Sixty specimens were collected from patients with ESCC to determine the possible role of CD276 in ESCC development and progression. First, we examined the expression of the CD276 gene in ESCC tissue samples, revealing that it was significantly upregulated in ESCC tissues compared with adjacent tissues (Figure 1A). This result was also verified by an analysis of TCGA data (Figure S1). Then, the levels of the CD276 protein in tumor tissues and non-tumor tissues were detected using IHC staining. By detecting the expression of CD276 in nontumor tissues and tumor tissues, we found CD276 to be underexpressed in the former but overexpressed in the latter (Figure 1B,C). We performed a survival analysis of all patients with ESCC for whom follow-up information was available, and survival curves were plotted. The overall survival times of patients with ESCC presenting high CD276 expression were short (Figure 1D). Disease-free survival (DFS) curves showed the same result (Figure 1E). These results consistently provide functional evidence that CD276 is upregulated in ESCC tissues and predicts a poor prognosis.

\section{CD276 was negatively correlated with CD $8+T$ cell infiltration in ESCC}

Immune cell infiltration is a key metric used to evaluate whether patients with tumors will benefit from immunotherapy and provides guidance in selecting a more suitable treatment for patients (14). Accordingly, we considered that CD276 may be a key regulator of cancer immunity. The correlation of CD276 expression and immune cell infiltration was thus analyzed using TIMER2.0. CD276 expression was significantly correlated with immune cell infiltration, particularly CD8 + T cell infiltration (Figure S2). Subsequently, an analysis of TCGA database was conducted. CD276 expression was negatively correlated with CD8+ T cell infiltration in ESCC, and positively correlated with the expression of $\mathrm{T}$ cell immunoglobulin mucin 3 (TIM-3), a marker of T cell exhaustion (Figure $2 A$ ). This finding was also confirmed by the results of the analysis of gene expression in clinical samples (Figure 2B). The colocalization of immunofluorescence staining was used to further validate this phenomenon (Figure 2C). Not surprisingly, no colocalization was observed between CD276 and CD8+ T cells. CD8+ T cell infiltration was rarely detected when the tumor cells expressed CD276 at high levels. Conversely, increased CD8+ T cell infiltration was observed around CD276-negative tumor cells. All of these findings indicate the regulatory role of CD276 in immune cell infiltration and its negative correlation with CD8+ T cells. Thus, CD276 may be a key regulator of CD8+ $\mathrm{T}$ cell infiltration in ESCC.

\section{CD276 promoted glycolysis and lactic acid accumulation}

The Warburg effect, a hallmark of malignancy, is the main pathway used to meet the demands for tumor growth and proliferation. Highly active cancer cells exert profound effects on the tumor microenvironment leading to hypoxia, nutrient depletion, and acidity (15). The role of CD276 in glucose metabolism was detected. First, cell surface CD276 expression on ESCC cell lines was detected using flow cytometry. As expected, most of the tested ESCC cell lines expressed CD276 at high levels. EC109 was used as a low-expression cell line in subsequent experiments (Figure $3 A$ ). The transfection of small interfering RNA (siRNA) oligonucleotides against CD276 into KYSE150 and KYSE510 cells was used to deplete CD276. Compared with the control (siNC) transfection, significant losses of CD276 were achieved. Overexpression of CD276 from a lentivirus also produced significant differences (Figure S3). Energy metabolism was determined with a Seahorse instrument. An evaluation of ECAR indicated that the cells with high CD276 expression generated more extracellular lactate (higher ECAR) than the cells with low expression of CD276 (Figure 3B). The basal ECAR was also high in cells with high CD276 expression (Figure 3C). Culture 
A

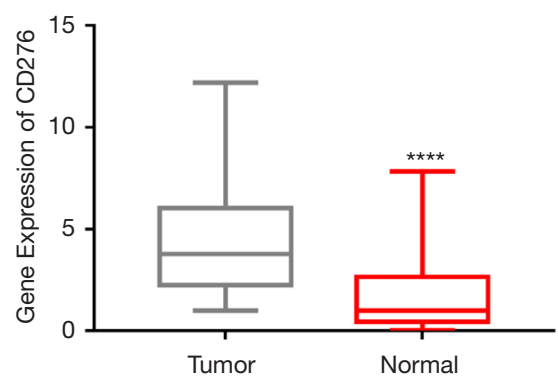

C

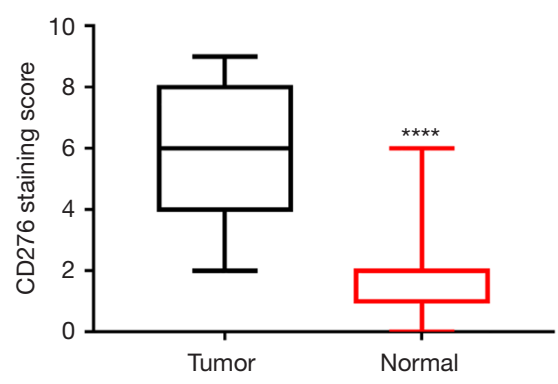

B

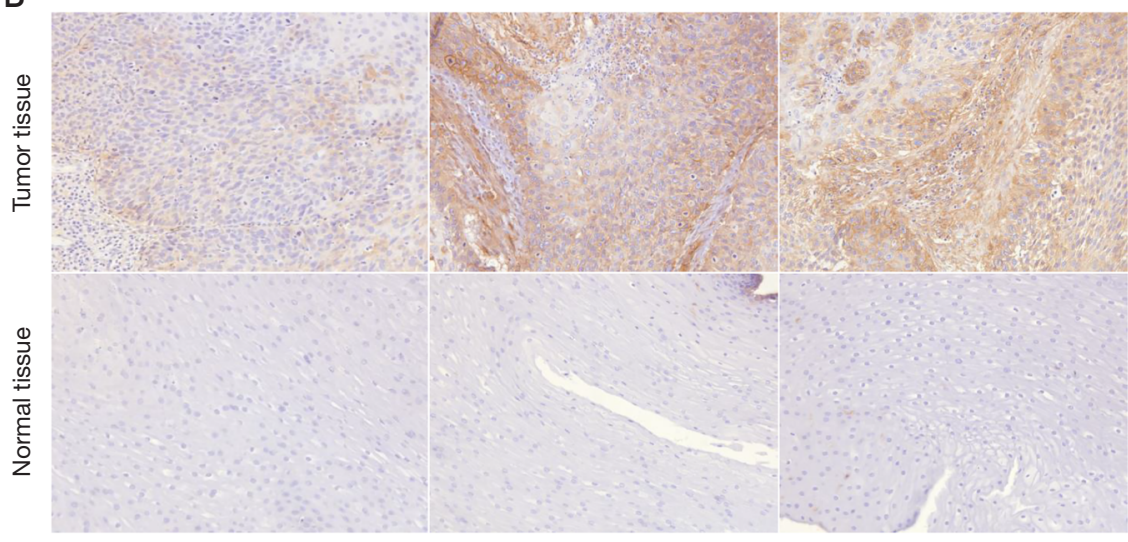

D

Overall Survival

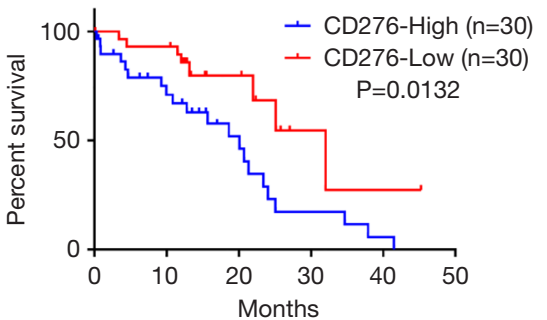

E

Disease-free Survival

Figure 1 CD276 was upregulated in ESCC tissues and predicted poor prognosis. (A) Relative expression levels of CD276 in ESCC and normal tissues were quantified using qRT-PCR. (B) IHC staining for CD276 in ESCC and normal tissues (200x). (C) IHC scores for CD276 in ESCC and normal tissues. (D) Kaplan-Meier survival analysis of patients' overall survival. (E) Kaplan-Meier survival analysis of patient s' disease-free survival. ${ }^{* * * *} \mathrm{P}<0.0001$. ESCC, esophageal squamous cell carcinoma; IHC, immunohistochemistry.

supernatants of stable overexpression or knockdown cell lines were harvested to detect the lactic acid level using a lactic acid detection kit. CD276 significantly stimulated lactate secretion (Figure 3D). Thus, we concluded that high CD276 expression promotes glycolytic metabolism and lactic acid accumulation in ESCC cells, suggesting possible mechanisms of immune evasion.

\section{PKM2 was upregulated in ESCC and positively correlated with CD276 expression}

The expression of key genes in glycolysis was analyzed in TCGA database to further investigate the mechanisms by which CD276 promotes glycolysis and lactic acid accumulation. Four genes (HK2, HK3, PFKP, and PKM2) were expressed at high levels in ESCC tissues (Figure 4A). A subsequent analysis was performed to investigate the correlation between the expression of CD276 and these four genes. The most obvious correlation was observed between CD276 and PKM2 (Figure 4B). This result strongly suggests that the effect of CD276 on promoting glycolytic metabolism and disease progression depended on PKM2. However, further experiments are necessary to validate our prediction. We detected the changes in the expression of genes involved in glycolysis in ESCC cells overexpressed or silenced for CD276. Among these nine glycolysisrelated genes, the most obvious change was observed in the expression of $P K M 2$, and this result is consistent with our previous analysis (Figure 4C). The same trend was observed for Ki67, a proliferation marker of tumor cells, suggesting that the increase in glycolysis induced by CD276 provides energy for cell proliferation. Immunohistochemical staining of 60 ESCC samples was performed, and the results were consistent with the qRT-PCR analysis (Figure $4 D, E$ ). Thus, we concluded that the glycolysis-promoting effect of CD276 depends on PKM2 expression. 

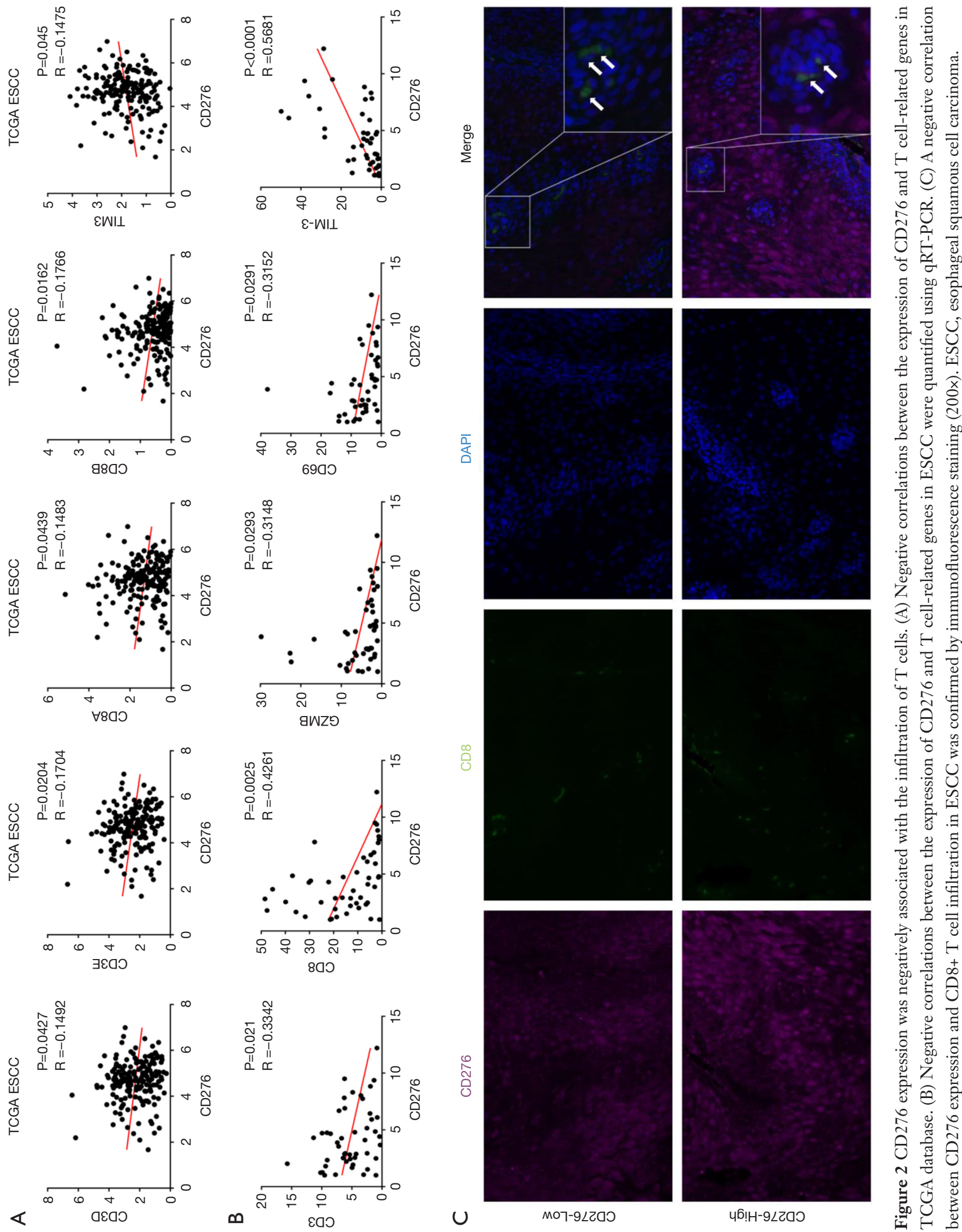

$\cup$

м07-9८20ว

4ढ़!H-9८Z০० 


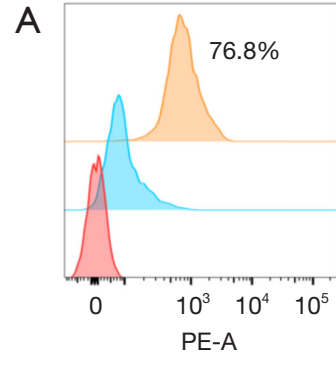

CD276

KYSE70

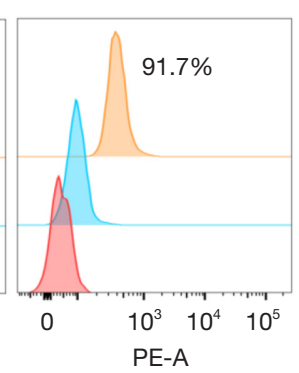

PE-A

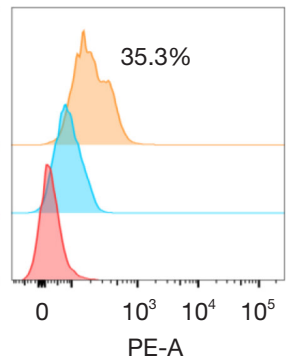

PE-A

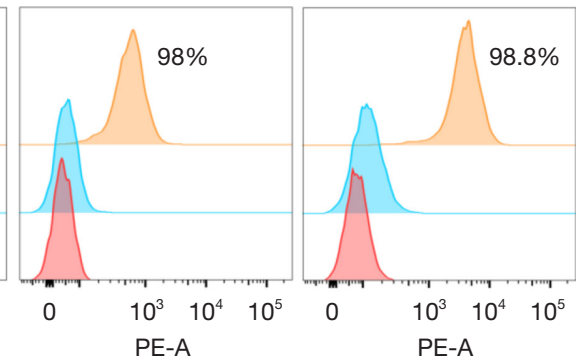

KYSE450

KYSE150

\section{B}
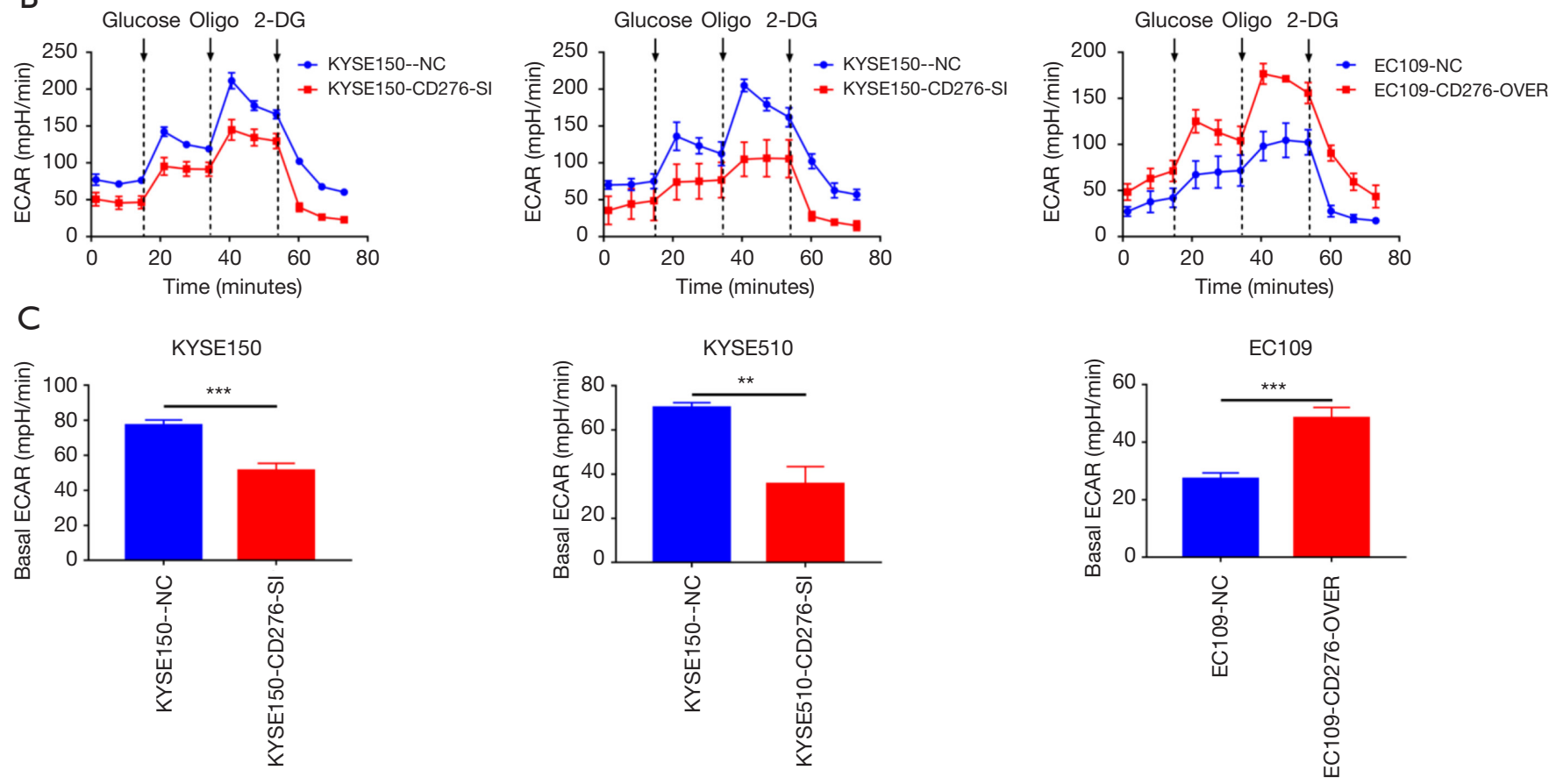

D

KYSE150

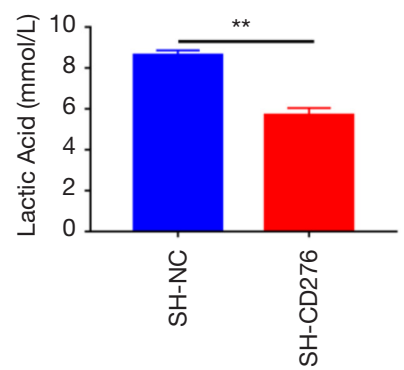

KYSE510

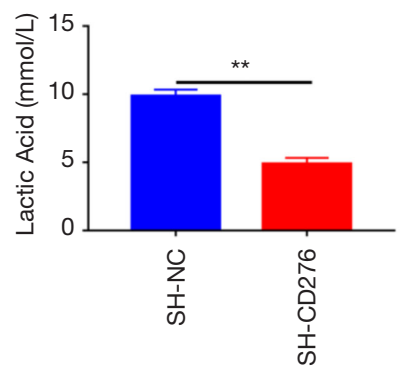

EC109

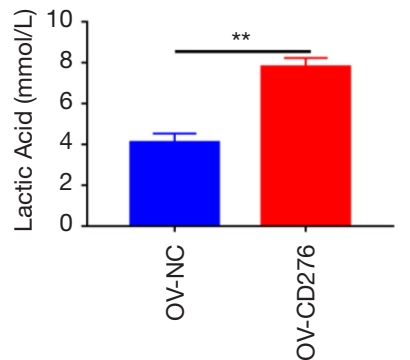

Figure 3 CD276 played an important role in promoting glycolysis and lactic acid accumulation in ESCC cell lines. (A) The expression of CD276 in ESCC cell lines was assessed using flow cytometry. (B) The ECAR of KESE150, KYSE510, and EC109 cells was analyzed when the expression of CD276 was changed. (C) Summary of the basal ECAR values shown in (B). (D) Lactic acid levels in culture supernatants were detected after 48 hours of incubation. ${ }^{* *} \mathrm{P}<0.01$, ${ }^{* * *} \mathrm{P}<0.001$. ESCC, esophageal squamous cell carcinoma. 

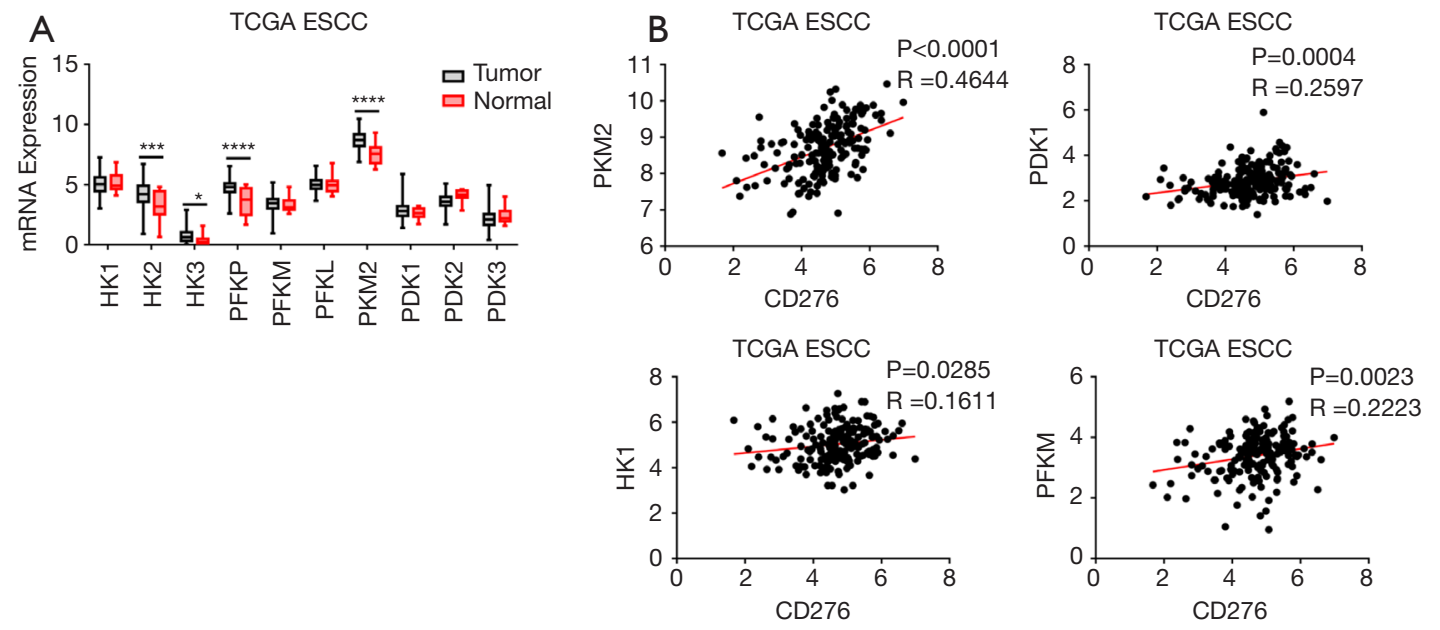

C
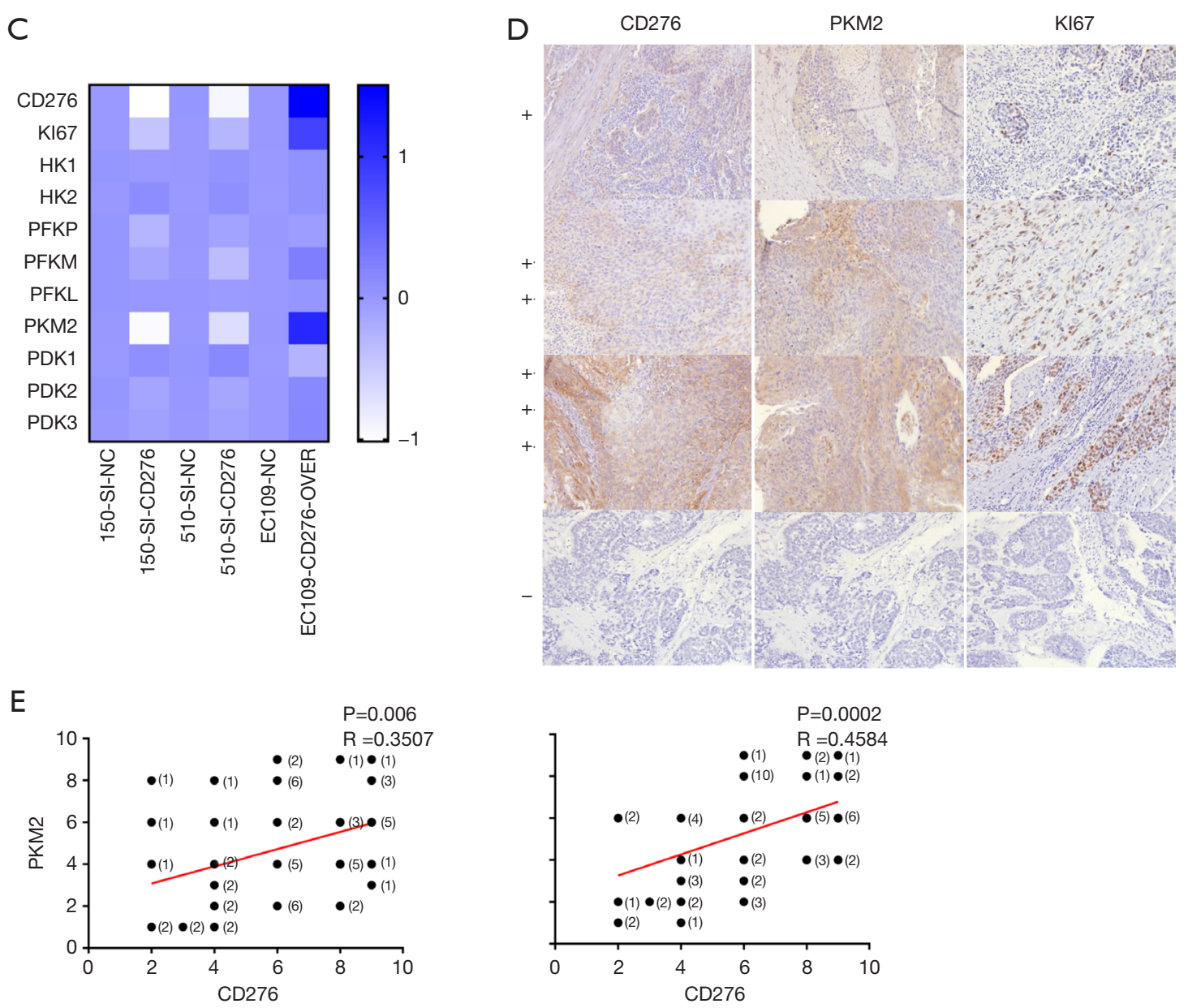

Figure 4 PKM2 was overexpressed in ESCC tissues and positively correlated with CD276 expression. (A) TCGA data were analyzed for the differential expression of critical glycolysis-related genes in ESCC and normal tissues. (B) The correlations between the expression of CD276 and PKM2, PDK1, HK1, and PFKM. (C) Heat maps showing the differential expression of critical glycolysis-related genes in ESCC cell lines when the expression of CD276 was changed. (D) IHC staining for CD276, PKM2, and Ki67 in ESCC tissues (200x). (E) Quantification of the staining for CD276, PKM2, and Ki67 in human ESCC tissues. Correlations between the expression of CD276, PKM2 and Ki67 were significant. ${ }^{* * *} \mathrm{P}<0.0001,{ }^{* * *} \mathrm{P}<0.001,{ }^{*} \mathrm{P}<0.05$. ESCC, esophageal squamous cell carcinoma. 


\section{STAT3/PKM2 mediated the tumor-promoting functions of CD276 in ESCC cells}

Activated signal transducer and activator of transcription 3 (STAT3) is required for tumor cell proliferation and survival, and CD276 has been reported to activate the JAK2/ STAT3 pathway $(9,16)$. We hypothesized that the potential mechanism by which PKM2 is regulated by CD276 relies on the phosphorylation of STAT3. Knockdown of CD276 in KYSE150 and KYSE510 cells reduced the activation of PKM2 and STAT3, while overexpression of CD276 increased the activation of these two molecules (Figure 5A,B). Then, the effect of PKM2 on CD276-induced cell proliferation was examined, and the results showed that full activation of PKM2 by TEPP-46 mimicked the tumorpromoting effects of CD276; moreover, inhibition of PKM2 by PKM2-IN-1 blocked CD276-induced cell growth (Figure 5C,D). Next, we further verified the role of STAT3 in this pathway. Small molecule inhibitors and STAT3 agonists were used to detected the effects of STAT3 activation. As expected, the small-molecule inhibitor C1889 offset the tumor-promoting effects of CD276. In contrast, the opposite result was observed when colivelin was used (Figure 5E,F). Taken together, the tumor-promoting functions of CD276 in ESCC at least partially rely on the activation of STAT3/PKM2.

\section{Tumor CD276 expression was associated with resistance to CAR-T therapy in ESCC}

CAR-T cell therapy, a method of immunotherapy for cancer, has shown great therapeutic potential (17). Lactic acid in the tumor microenvironment diminishes cholesterol synthesis and interferon gamma (IFN- $\gamma$ ) production by $\mathrm{T}$ and natural killer (NK) cells $(18,19)$. Lactic acid accumulation induced by CD276 may be a barrier for CAR-T cell therapy. We subcutaneously injected (s.c.) the Luc-transduced KYSE150 tumor cell line (HER2 positive, Figure S4A) into NOD/SCID/gamma(c) (null) (NSG) mice to evaluate the role of CD276 on CAR-T cells therapy in vivo. Seven days later, mice were intravenously (i.v.) infused with CAR-HER2-T (Figure S4B,C) cells or mock T cells via a tail vein injection (Figure $6 A$ ). Bioluminescent imaging was used to detect tumor signals. First, the tumors with high CD276 expression displayed more rapid progression than the tumors with low CD276 expression. Then, CARHER2-T cells, but not mock $T$ cells, showed cytotoxic activity against antigen-positive tumor cells. Complete tumor regression was only observed in the CAR-T treatment group with low CD276 expression in tumor cells (Figure 6B,C). This trend is also reflected in the differences in the survival of mice in the CAR-HER2-T group: CD276 low expression mice received an obvious improvement in survival time (Figure 6D). Subsequently, tumor tissues were removed from mice, and the tumor volume and weight were analyzed (Figure 6E,F). Compared with the other groups, the combination of CAR-HER2-T cell infusion and the knockdown of CD276 significantly inhibited the growth of xenografts.

\section{Discussion}

CD276 is an important immune checkpoint molecule that is abnormally expressed in multiple tumor types (20). The mechanism by which CD276 promotes tumor progression has been reported, and one of the major oncogenic signaling pathways of CD276 is the JAK2/STAT3 signaling pathway (21). CD276 contributes to tumor progression by regulating related molecules in the JAK2/STAT3 pathway in several types of cancer $(22,23)$. Here, we identified that the oncogenic role of CD276 in ESCC was the in vitro and in vivo promotion of ESCC cell proliferation and resistance to $\mathrm{T}$ cell therapy. The molecular mechanism underlying these effects of CD276 involves the induction of glycolysis through the expression of PKM2 and subsequent lactic acid accumulation.

As a promising therapeutic target, CD276 was expressed at high levels in tumor cells and at low levels in normal tissues. Relying on multiple mechanisms, CD276 may be involved in the proliferation and migration of cancer cells and in tumor progression. Overexpression of CD276 was reported to promote tumor stemness and induce drug resistance in cancer cells (24). As shown in our study, $\mathrm{CD} 276$ is an emerging pro-oncogenic factor because of its high expression in ESCC. High CD276 expression in tumor tissues was associated with a significantly worse prognosis. The same trend was observed for DFS. This potential correlation has attracted our interest and further research is urgently needed.

The Warburg effect is the characteristic and main metabolic pathway that provides steady energy for the growth of tumor cells (25). In our study, CD276 regulated the expression of PKM2, a rate-limiting enzyme in the final step of glycolysis. Numerous studies have reported a key function for PKM2 in glycolysis. The enzymatic 
A

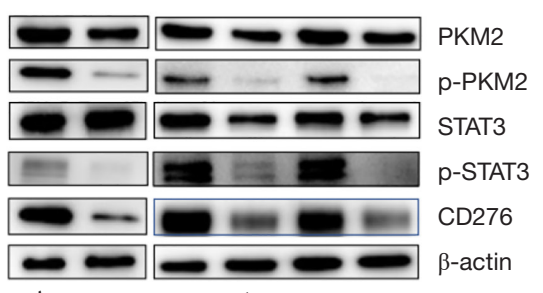

B

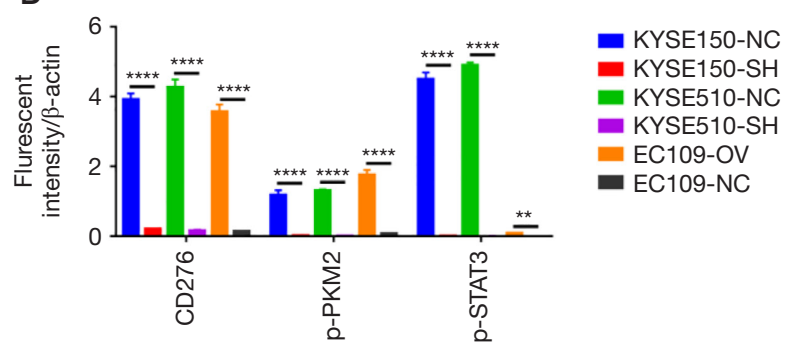

C

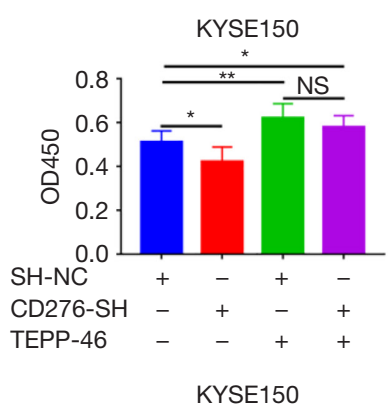

D

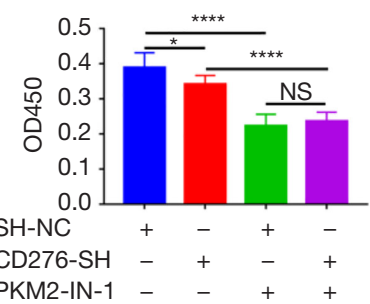

E
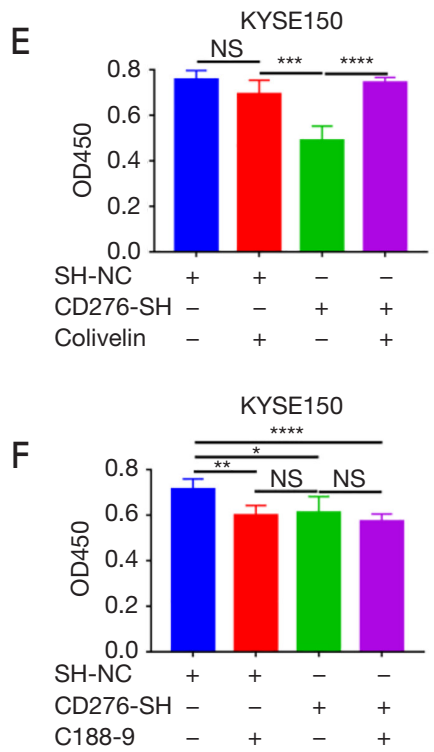
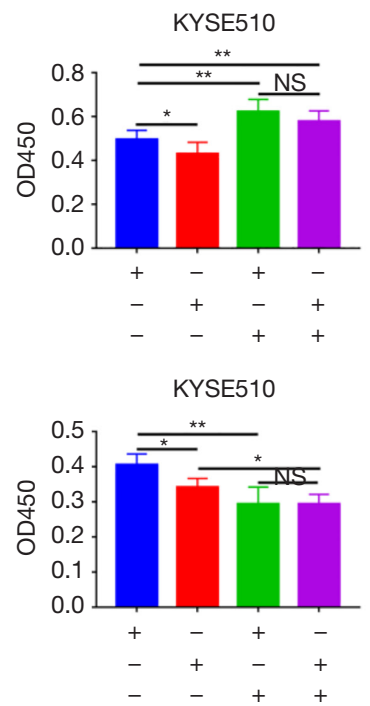

KYSE510
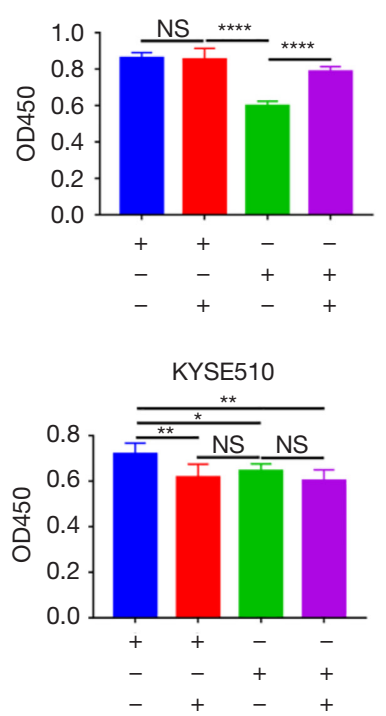
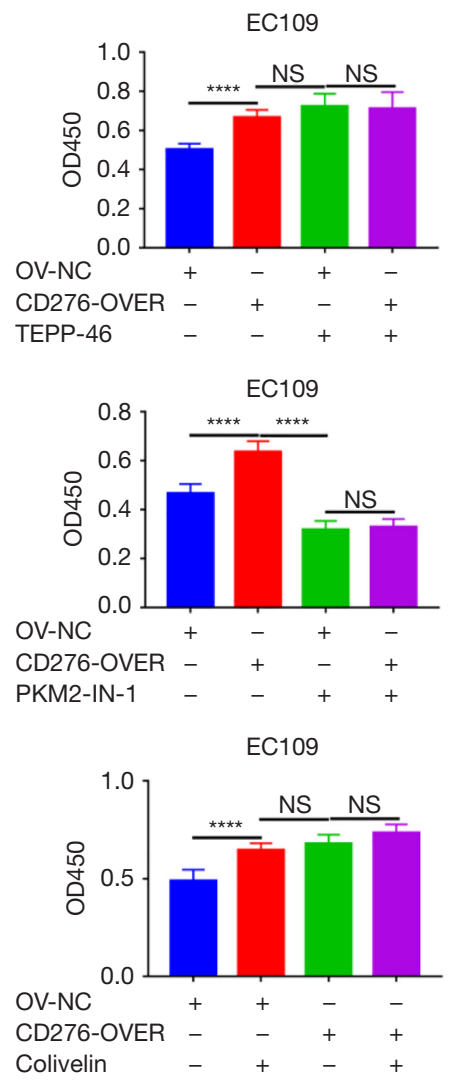

EC109

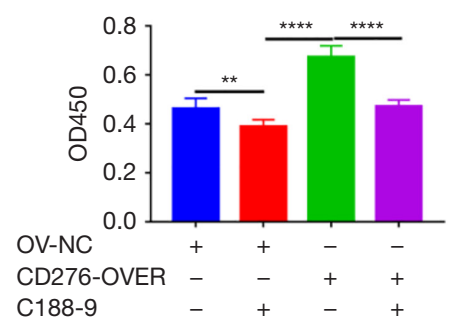

Figure 5 CD276 activated STAT3 signalling and promoted glycolysis via PKM2. (A) CD276 induced STAT3 and PKM2 phosphorylation in ESCC cell lines. (B) Grey value analysis of western blotting results. (C,D) The increased viability of CD276-overexpressing ESCC cells was abolished by PKM2 inhibition, whereas the decrease in the viability of CD276-silenced ESCC cells was restored by PKM2 activation. (E,F) The increased viability of CD276-overexpressing ESCC cells was abolished by STAT3 inhibition, whereas the decrease in the viability of CD276-silenced ESCC cells was restored by STAT3 activation. ${ }^{* * *} \mathrm{P}<0.0001,{ }^{* * *} \mathrm{P}<0.001,{ }^{* *} \mathrm{P}<0.01,{ }^{*} \mathrm{P}<0.05$. ESCC, esophageal squamous cell carcinoma. 
A KYSE150-Luc T cells (i.v.) IVIS imaging (s.c.) $1^{*} 10^{6} \quad 1^{*} 10^{6}$

$\underset{\text { Day0 }}{\downarrow} \quad$ Day7

B

Mock-T

D7

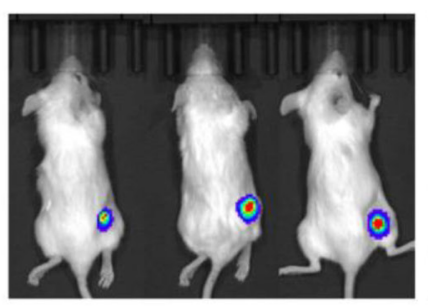

CD276-SH

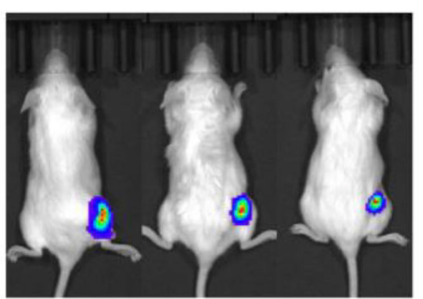

Her2.CAR-T
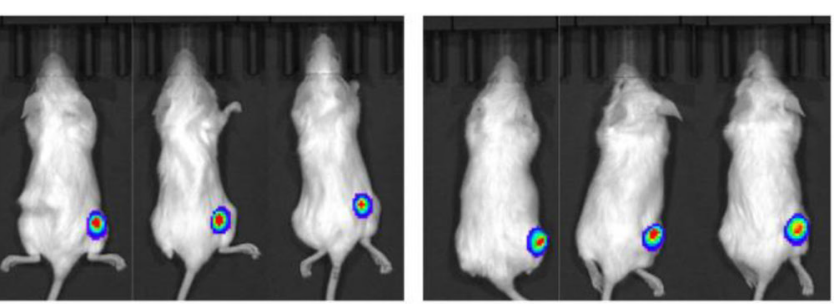

D21
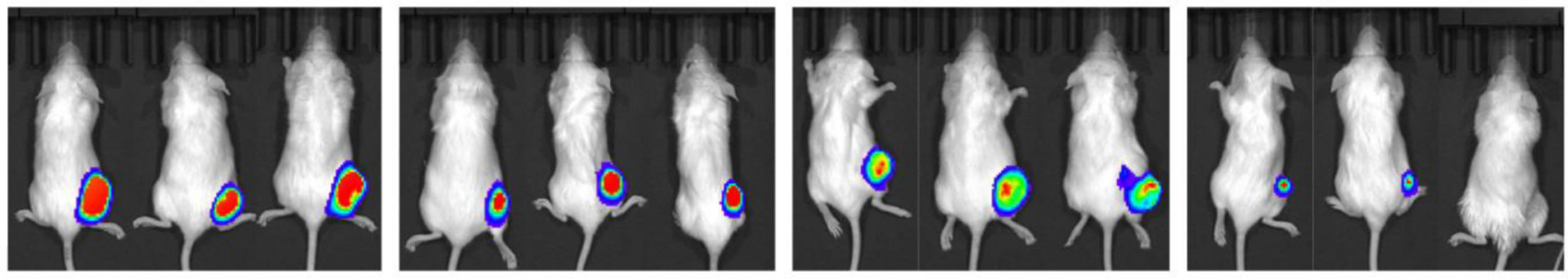

C

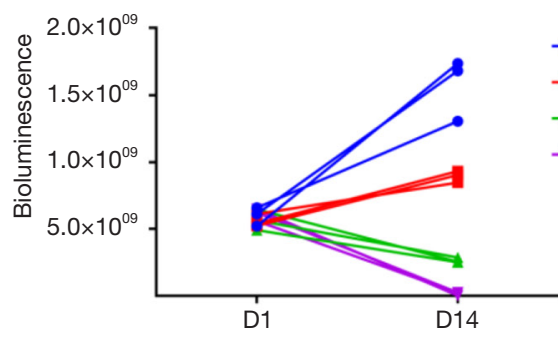

$\rightarrow$ Mock-T.CD276-High

- Mock-T.CD276-Low

- CAR-Her2.CD276-High

* CAR-Her2.CD276-Low

Days (post-CAR-T infusion)

E

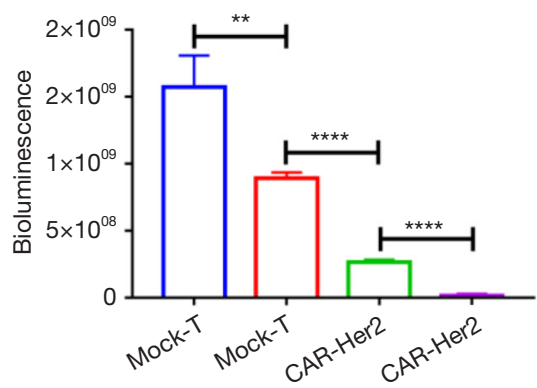

CD276 High Low High Low

D

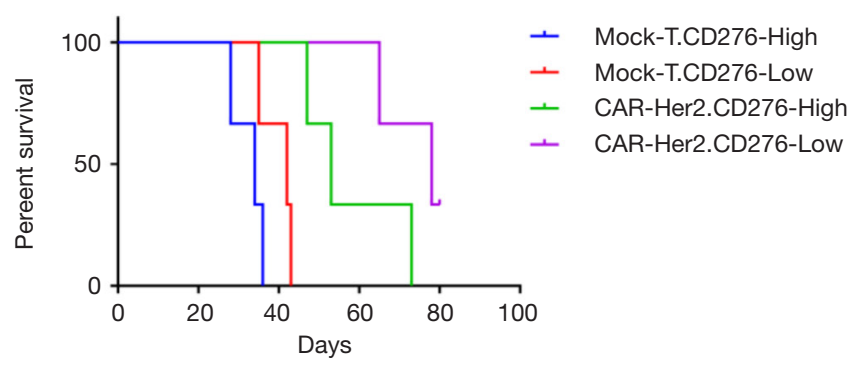

F

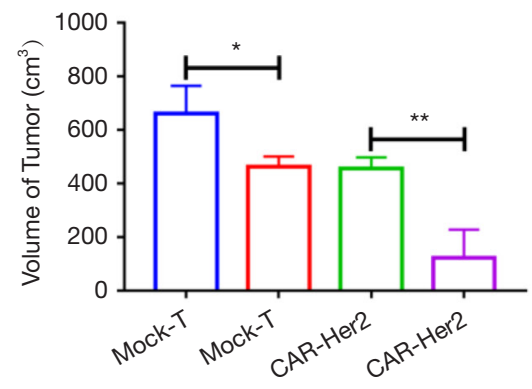

CD276 High Low High Low

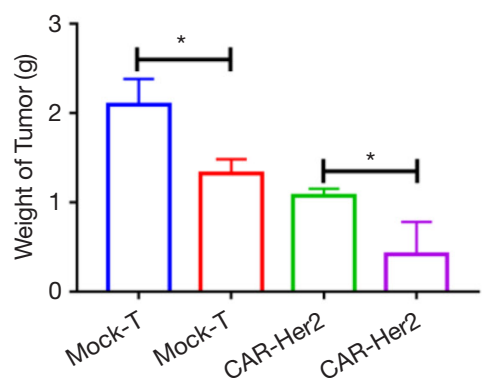

CD276 High Low High Low

Figure 6 High CD276 expression significantly promoted tumor progression and resistance to CAR-T cells therapy. (A) Schematic of the adoptive transfer experiment using NOG mice. (B,C) Images and total luminescence were collected and analyzed. (D) Survival curve of the mice shown in (B). (E) Statistical analysis of the final tumor volumes. (F) Statistical analysis of the final tumor weights. ${ }^{* * * *} \mathrm{P}<0.0001$, ${ }^{*} \mathrm{P}<0.01,{ }^{*} \mathrm{P}<0.05$. CAR-T, chimeric antigen receptor $\mathrm{T}$ cell. 
reaction catalyzed by PKM2 provides the necessary energy for tumor cell growth and substrates for the synthesis of macromolecules (26). This study is the first to report the regulatory function of CD276 in glycolysis. The significant correlation of CD276 expression and PKM2 in ESCC was confirmed by IHC staining and an analysis of TCGA data. The expression of PKM2 was upregulated in ESCC cell lines when CD276 was overexpressed. Furthermore, glycolysis and lactic acid accumulation were increased in tumor cells, while CD276 knockdown exerted the opposite effect. To the best of our knowledge, this is the first study to report the mechanism by which CD276 promotes ESCC cell proliferation. However, the function of CD276 was mediated by PKM2. Based on these data, CD276/PKM2 is a crucial pathway involved in the progression of ESCC.

Lactic acid is increasingly considered an immunomodulatory factor in tumors. The accumulation of lactic acid in the tumor microenvironment has been shown to promote the M2-like phenotype of tumor-associated macrophages, along with the upregulation of vascular endothelial growth factor and arginase 1 (27). Inhibition of glycolysis in cancer cells limits myeloid-derived suppressor cell development and the secretion of granulocyte colonystimulating factor and granulocyte-macrophage colonystimulating factor (28). Moreover, tumors with increased lactic acid production have been shown to reduce infiltration of IFN- $\gamma$-producing $\mathrm{T}$ and NK cells (29). As shown in the present study, CD276 is a potent inhibitor of the function and survival of $\mathrm{T}$ cells, and can contribute to the immune escape of tumor cells due to the secretion of lactic acid. It was also found that knockdown of CD276 could promote the antitumor activity of CAR-T cell therapy in vivo. Thus, we concluded that CD276 overexpression could significantly promote tumor progression and resistance to $\mathrm{T}$ cell therapy. Overall, our experiments showed CD276 to be a cancer-promoting and immunosuppressive factor in ESCC.

\section{Conclusions}

This study demonstrated that CD276 is significantly overexpressed in ESCC tissues and cells, and its high expression is significantly related to the poor prognosis of patients. Our study revealed new regulatory mechanisms of CD276 in tumors: CD276 regulates glycolysis through a mechanism that relies on the expression of PKM2 and subsequent lactic acid accumulation. Tumors with increased lactic acid production show resistance to CAR-T cell therapy. Taken together, our results indicate that CD276 may represent as a novel target for immunotherapy resistance.

\section{Acknowledgments}

We would like to thank the patients and their families for their signed informed consent for use of samples in clinical experiments.

Funding: None.

\section{Footnote}

Reporting Checklist: The authors have completed the MDAR reporting checklist. Available at http://dx.doi.org/10.21037/ jgo-21-50

Data Sharing Statement: Available at http://dx.doi. org/10.21037/jgo-21-50

Conflicts of Interest: All authors have completed the ICMJE uniform disclosure form (available at http://dx.doi. org/10.21037/jgo-21-50). The authors have no conflicts of interest to declare.

Etbics Statement: The authors are accountable for all aspects of the work in ensuring that questions related to the accuracy or integrity of any part of the work are appropriately investigated and resolved. The research protocol was reviewed and approved by the Ethics Committee of Zhengzhou University, and informed consent was obtained from all participants included in the study, in agreement with institutional guidelines (No. 2016-lw-107). This study conformed to the provisions of the Declaration of Helsinki (as revised in 2013). All xenograft mouse model experiments were performed in the Henan Key Laboratory for Pharmacology of Liver Diseases, and the animal experimentation certificate was approved by the ethics committee of Henan Key Laboratory for Pharmacology of Liver Diseases (approval number: 2019-41). All animal experiments were performed in accordance with the guidelines of ethics committee of Henan Key Laboratory for Pharmacology of Liver Diseases for animal use and care.

Open Access Statement: This is an Open Access article distributed in accordance with the Creative Commons Attribution-NonCommercial-NoDerivs 4.0 International License (CC BY-NC-ND 4.0), which permits the noncommercial replication and distribution of the article with 
the strict proviso that no changes or edits are made and the original work is properly cited (including links to both the formal publication through the relevant DOI and the license). See: https://creativecommons.org/licenses/by-nc-nd/4.0/.

\section{References}

1. Zhao Z, Wen Y, Liao D, et al. Single-Agent Versus Double-Agent Chemotherapy in Concurrent Chemoradiotherapy for Esophageal Squamous Cell Carcinoma: Prospective, Randomized, Multicenter Phase II Clinical Trial. Oncologist 2020;25:e1900-8.

2. Zhou X, Li Y, Wang W, et al. Regulation of Hippo/ YAP signaling and Esophageal Squamous Carcinoma progression by an E3 ubiquitin ligase PARK2 . Theranostics 2020;10:9443-57.

3. Barros LRC, Souza-Santos PT, Pretti MAM, et al. High infiltration of B cells in tertiary lymphoid structures, TCR oligoclonality, and neoantigens are part of esophageal squamous cell carcinoma microenvironment. J Leukoc Biol 2020;108:1307-18.

4. Yang S, Wei W, Zhao Q. B7-H3, a checkpoint molecule, as a target for cancer immunotherapy. Int J Biol Sci 2020;16:1767-73.

5. Gootjes EC, Kraan J, Buffart TE, et al. CD276-Positive Circulating Endothelial Cells Do Not Predict Response to Systemic Therapy in Advanced Colorectal Cancer. Cells 2020;9:124.

6. Maachani UB, Tosi U, Pisapia DJ, et al. B7-H3 as a Prognostic Biomarker and Therapeutic Target in Pediatric central nervous system Tumors. Transl Oncol 2020;13:365-71.

7. MacGregor HL, Sayad A, Elia A, et al. High expression of $\mathrm{B} 7-\mathrm{H} 3$ on stromal cells defines tumor and stromal compartments in epithelial ovarian cancer and is associated with limited immune activation. J Immunother Cancer 2019;7:357.

8. Schneider T, Hoffmann H, Dienemann H, et al. Nonsmall cell lung cancer induces an immunosuppressive phenotype of dendritic cells in tumor microenvironment by upregulating B7-H3. J Thorac Oncol 2011;6:1162-8.

9. Lin L, Cao L, Liu Y, et al. B7-H3 promotes multiple myeloma cell survival and proliferation by ROS-dependent activation of Src/STAT3 and c-Cbl-mediated degradation of SOCS3. Leukemia 2019;33:1475-86.

10. Theruvath J, Sotillo E, Mount CW, et al. Locoregionally administered B7-H3-targeted CAR T cells for treatment of atypical teratoid/rhabdoid tumors. Nat Med
2020;26:712-9.

11. Du H, Hirabayashi K, Ahn S, et al. Antitumor Responses in the Absence of Toxicity in Solid Tumors by Targeting B7-H3 via Chimeric Antigen Receptor T Cells. Cancer Cell 2019;35:221-37.e8.

12. Li T, Fu J, Zeng Z, et al. TIMER2.0 for analysis of tumor-infiltrating immune cells. Nucleic Acids Res 2020;48:W509-14.

13. Bubner B, Baldwin IT. Use of real-time PCR for determining copy number and zygosity in transgenic plants. Plant Cell Rep 2004;23:263-71.

14. Zhang K, Zhang L, Mi Y, et al. A ceRNA network and a potential regulatory axis in gastric cancer with different degrees of immune cell infiltration. Cancer Sci 2020;111:4041-50.

15. Leone RD, Powell JD. Metabolism of immune cells in cancer. Nat Rev Cancer 2020;20:516-31.

16. Kang FB, Wang L, Jia HC, et al. B7-H3 promotes aggression and invasion of hepatocellular carcinoma by targeting epithelial-to-mesenchymal transition via JAK2/STAT3/Slug signaling pathway. Cancer Cell Int 2015;15:45.

17. Wu W, Zhou Q, Masubuchi T, et al. Multiple Signaling Roles of CD3epsilon and Its Application in CAR-T Cell Therapy. Cell 2020;182:855-871.e23.

18. Fu S, He K, Tian C, et al. Impaired lipid biosynthesis hinders anti-tumor efficacy of intratumoral iNKT cells. Nat Commun 2020;11:438.

19. Brand A, Singer K, Koehl GE, et al. LDHA-Associated Lactic Acid Production Blunts Tumor Immunosurveillance by T and NK Cells. Cell Metab 2016;24:657-71.

20. Kanchan RK, Perumal N, Atri P, et al. MiR-1253 exerts tumor-suppressive effects in medulloblastoma via inhibition of CDK6 and CD276 (B7-H3). Brain Pathol 2020;30:732-45.

21. Chen X, Meng X, Foley NM, et al. Activation of the TLR2-mediated downstream signaling pathways NF$\kappa \mathrm{B}$ and MAPK is responsible for $\mathrm{B} 7-\mathrm{H} 3$-augmented inflammatory response during $\mathrm{S}$. pneumoniae infection. J Neuroimmunol 2017;310:82-90.

22. Zhang J, Liu L, Han S, et al. B7-H3 is related to tumor progression in ovarian cancer. Oncol Rep 2017;38:2426-34.

23. Fan TF, Deng WW, Bu LL, et al. B7-H3 regulates migration and invasion in salivary gland adenoid cystic carcinoma via the JAK2/STAT3 signaling pathway. Am J Transl Res 2017;9:1369-80.

24. Liu Z, Zhang W, Phillips JB, et al. Immunoregulatory protein $\mathrm{B} 7-\mathrm{H} 3$ regulates cancer stem cell enrichment and 
drug resistance through MVP-mediated MEK activation. Oncogene 2019;38:88-102.

25. Morita M, Kanasaki K. Sodium-glucose cotransporter-2 inhibitors for diabetic kidney disease: Targeting Warburg effects in proximal tubular cells. Diabetes Metab 2020;46:353-361.

26. Liu B, Song M, Qin H, et al. Phosphoribosyl Pyrophosphate Amidotransferase Promotes the Progression of Thyroid Cancer via Regulating Pyruvate Kinase M2. Onco Targets Ther 2020;13:7629-39.

27. Colegio OR, Chu NQ, Szabo AL, et al. Functional

Cite this article as: Yue G, Tang J, Zhang L, Niu H, Li H, Luo S. CD276 suppresses CAR-T cell function by promoting tumor cell glycolysis in esophageal squamous cell carcinoma. J Gastrointest Oncol 2021;12(1):38-51. doi: 10.21037/jgo-21-50 polarization of tumour-associated macrophages by tumourderived lactic acid. Nature 2014;513:559-63.

28. Li W, Tanikawa T, Kryczek I, et al. Aerobic Glycolysis Controls Myeloid-Derived Suppressor Cells and Tumor Immunity via a Specific CEBPB Isoform in TripleNegative Breast Cancer. Cell Metab 2018;28:87-103.e6.

29. Scott KE, Cleveland JL. Lactate Wreaks Havoc on TumorInfiltrating T and NK Cells. Cell Metab 2016;24:649-50.

(English Language Editor: J. Gray) 


\section{Supplementary}

Table S1 Primers used for qRT-PCT

\begin{tabular}{lll}
\hline Gene & Forward (5'-3') & Reverse (5'-3') \\
\hline CD276 & CTG GCTTTCGTGTGCTGGAGAA & GCTG TCAGAGTGTTCAGAGGC \\
GAPDH & CTGGGCTACACTGAGCACC & AAGTGGTCGTTGAGGGCAATG \\
PKM2 & ATGTCGAAGCCCATAGTGAA & TGGGTGGTGAATCAATGTCCA \\
MKI67 & CTGACCCTGATGAGAGTGAGGGA & ACTCTGTAGGGTCGAGCAGG \\
CD3D & ACTGGCTACCCTTCTCTCG & CCGTTCCCTCTACCCATGTGA \\
CD8A & ATGGCCTTACCAGTGACCG & AGGTTCCAGGTCCGATCCAG \\
GZMB & CCCTGGGAAAACACTCACACA & GCACAACTCAATGGTACTGTCG \\
CD69 & ATTGTCCAGGCCAATACACATT & CCTCTCTACCTGCGTATCGTTT \\
TIM3 & CTGCTGCTACTACTTACAAGGTC & GCAGGGCAGATAGGCATTCT \\
HK1 & GCTCTCCGATGAAACTCTCATAG & GGACCTTACGAATGTTGGCAA \\
HK2 & GAGCCACCACTCACCCTACT & CCAGGCATTCGGCAATGTG \\
PFKP & GCATGGGTATCTACGTGGGG & CTCTGCGATGTTTGAGCCTC \\
PFKM & GGTGCCCGTGTCTTCTTGT & AAGCATCATCGAAACGCTCTC \\
PFKL & GCTGGGCGGCACTATCATT & TCAGGTGCGAGTAGGTCCG \\
PDK1 & CTGTGATACGGATCAGAAACCG & TCCACCAAACAATAAAGAGTGCT \\
PDK2 & ATGAAAGAGATCAACCTGCTTCC & GGCTCTGGACATACCAGCTC \\
PDK3 & CGCTCTCCATCAAACAATTCCT & CCACTGAAGGGCGGTTAAGTA \\
\hline
\end{tabular}

TCGA ESCC

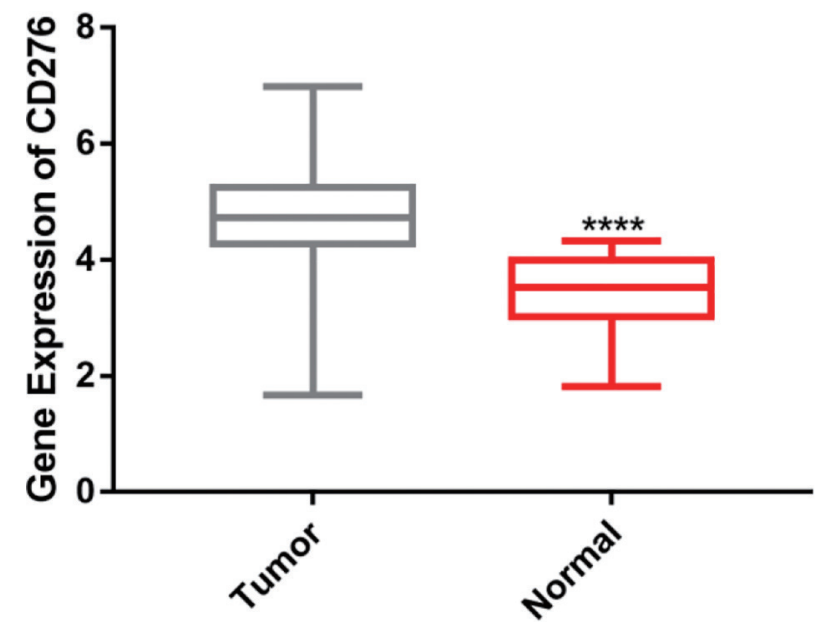

Figure S1 Relative levels of CD276 expression in ESCC and normal tissues from TCGA data were analyzed. ${ }^{* * *} \mathrm{P}<0.0001$. ESCC, esophageal squamous cell carcinoma. 

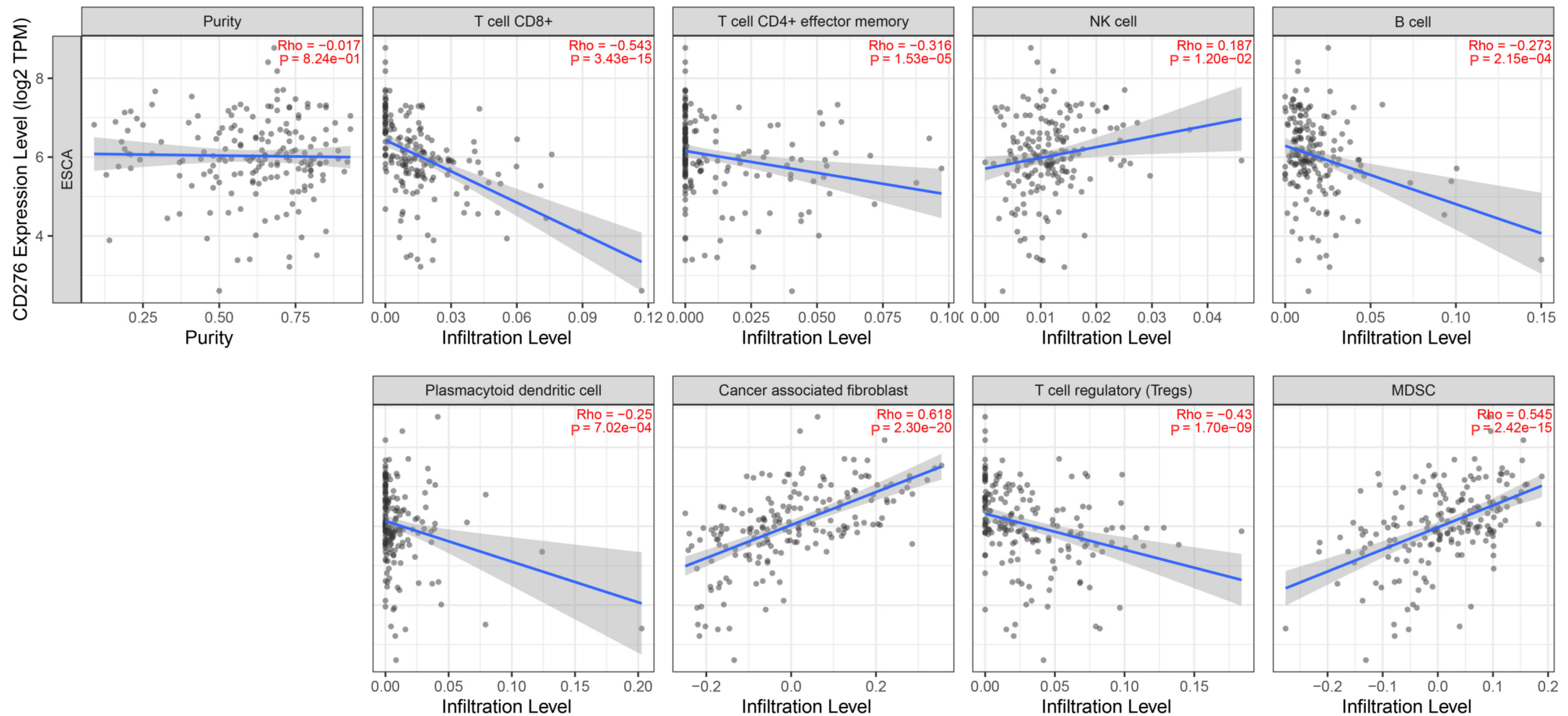

Figure S2 The correlation between CD276 expression and immune cell infiltration was analyzed by TIMER2.0. 
KYSE150

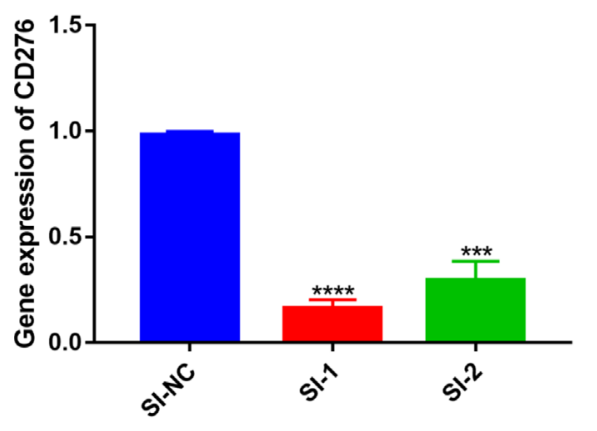

KYSE510

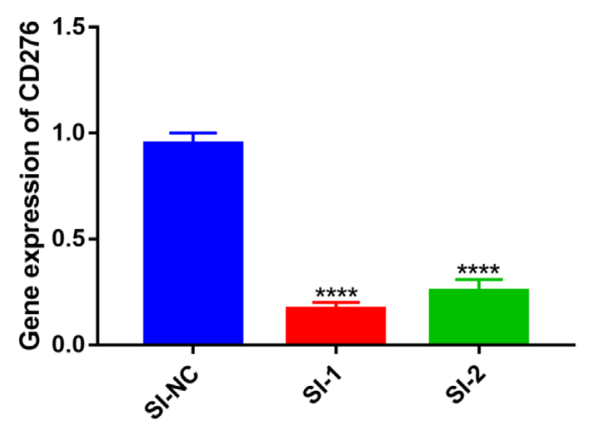

EC109

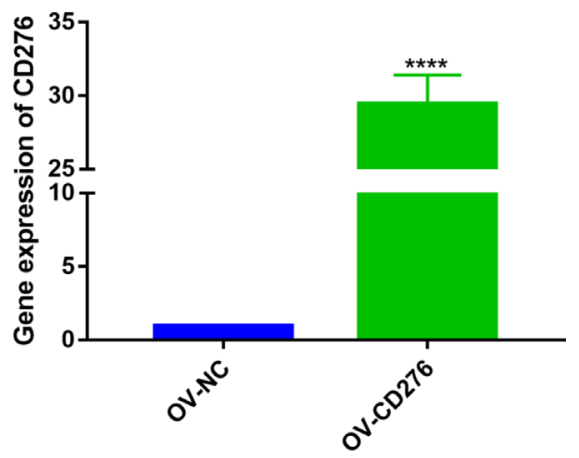

Figure S3 Efficiency of CD276 knockdown and overexpression in ESCC cell lines. ESCC, esophageal squamous cell carcinoma. ${ }^{* * * *} \mathrm{P}<0.0001,{ }^{* * *} \mathrm{P}<0.001$.

\section{A}

B

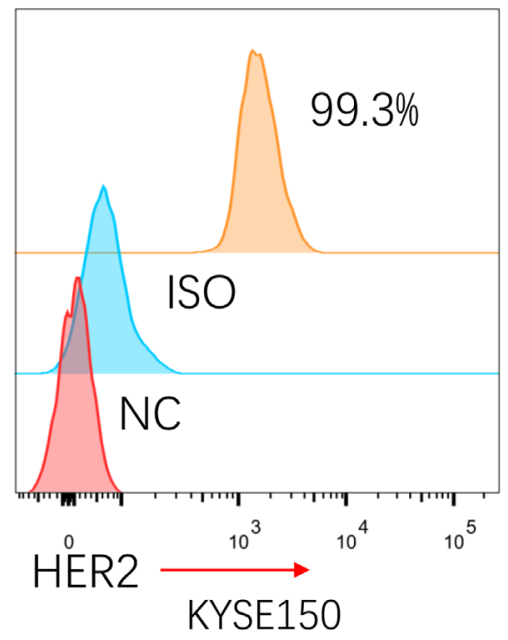

C

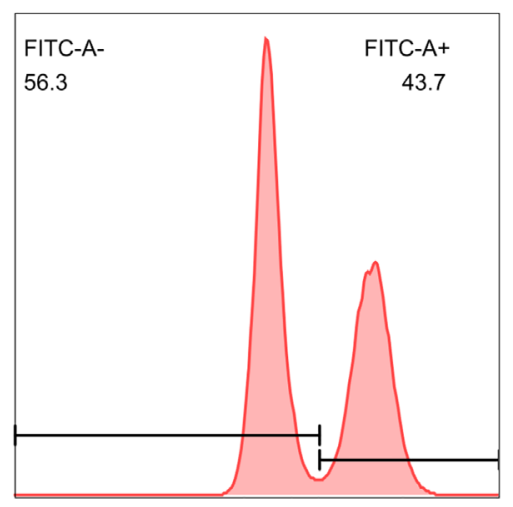

GFP

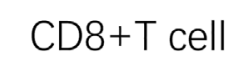

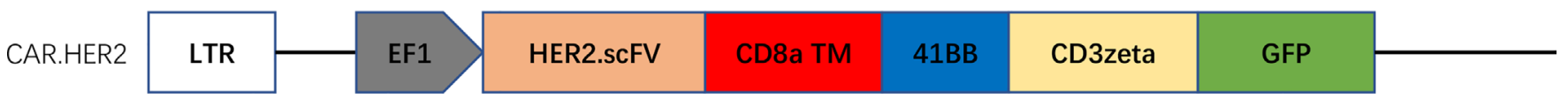

Figure S4 Antigen detection and construction of CAR T cells. (A) Expression of HER2 in the KYSE150 cell line. (B) Schematic representation of the retroviral vector encoding CAR-HER2. (C) Flow cytometry histograms showing CAR-HER2 expression in activated $\mathrm{T}$ cells. CAR-T, chimeric antigen receptor $\mathrm{T}$ cell. 